\title{
EFFECT OF THE INTERACTIONS BETWEEN MOISTURE CONTENT, SEED STORAGE TIME AND USE OF PESTICIDES ON SEED GERMINATION, VIABILITY AND VIABILITY INDICATORS OF WHEAT CROP
}

\section{Mohammad Ali JAHANBIN, Hasan Hüseyin GEÇİT and Saime ÜNVER İKİNCİKARAKAYA}

Department of Agronomy and Plant breeding Ankara of University Ankara Turkey

Received - April 20, 2016; Revision - May 25, 2016; Accepted - June 28, 2016

Available Online - June 30, 2016

DOI: http://dx.doi.org/10.18006/2016.4(4).406.413

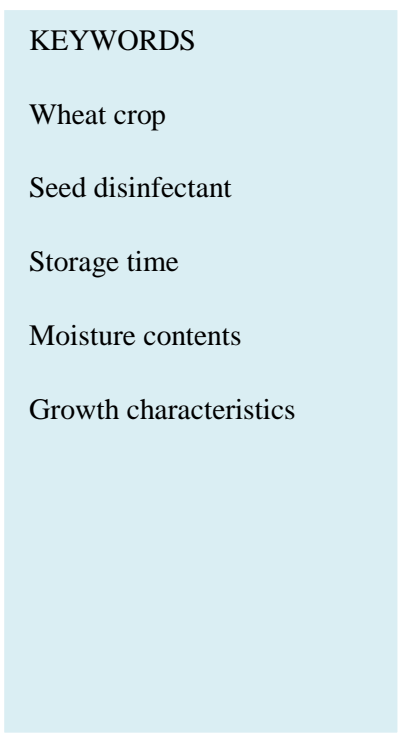

\begin{abstract}
In order to determine the effect of moisture content, storage time and pesticide (fungicide \& insecticide) application in wheat variety Bezostaja -1, an experiment was conducted in Split-Split plot, randomized complete block design with three replications for two years at Ankara University. The main plots have three seed moisture levels viz. standard, standard $+1.5 \%$ and standard +3 percent at the time of disinfect application. The Split plots (sub plot) have treatments like seed storage time viz: 0, 1, 2 and 14 months and sub- sub plot was pesticide application and types and pesticide level. In this study two pesticides viz. Diaconazole (\% 1), Chlorpyrifos - ethyl (\% $25 \mathrm{w}$ p) was used either individually or in combination. Various wheat characters which studied in present study are Coefficient of Velocity of Germination (CVG), Germination Index (GI), Germination Rate Index (GRI), Coleoptile length, Root length, Fresh weight and Dry weight. Result of study revealed that interactions between pesticides, seed storage and seed moisture content have significant effect $(1 \%)$ on the various studied growth characteristics such as CVG, GI, GRI, Shoot length, root length, fresh weight and dry weight. Further, it was reported that the traits such as seed germination percentage, germination index, fresh weight and root length have very higher efficiency in the maintenance of seed for one to two months at the application of anti-fungal toxins with $9.5 \%$ moisture Content. While minimum storage efficiency was reported when combination of pesticides (fungicide + insecticide) were applied at $12.5 \%$ moisture.
\end{abstract}

* Corresponding author

E-mail: ma_jahan51@yahoo.com (Mohammad Ali JAHANBIN)

Peer review under responsibility of Journal of Experimental Biology and Agricultural Sciences.

Production and Hosting by Horizon Publisher India [HPI] (http://www.horizonpublisherindia.in/).

All rights reserved.
All the article published by Journal of Experimental Biology and Agricultural Sciences is licensed under a Creative Commons Attribution-NonCommercial 4.0 International License Based on a work at www.jebas.org.

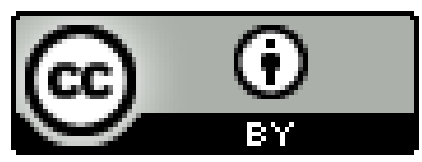




\section{Introduction}

Wheat is one of the most important cereal crops in the world, together with rice and maize; it provides more than $60 \%$ of the calories and proteins of human nutrition (Nord et al., 2009). From last few decades despite of increasing crop yield intensive agricultural and monoculture systems adversely affected its production. Although, use of fertilizers, herbicides, pesticides and modified seeds have significant effect on the crop production but according to world pesticides trade continues use of stable insecticides and pesticides induced resistance in the pest or insect and losses resulting from pest damage continuously increases (Gliessman, 2000). Further, although use of improved seeds and cultural practices can be a better option of pesticides but farmers still rely on the use of pesticides and they continuously increased dose of pesticide for getting more production this operation not only increase the cost of farming but also cause health and environmental hazards (Clark \& Scott, 1982). This excess use of pesticides also responsible for the loss of biodiversity by destroying soil micro-organisms and other organisms, sometime these pesticides reached to the ground water and also contaminate (Lu \& Terry, 1995).

Among various factors which influencing crop productivity, seed quality and seed moisture contents are most important one. According to Dupriez \& De Leener (1989) best quality seed have high germination capacity and resultant seedlings are vigor than the poor quality seeds. Seed quality is a condition under which mother plants were raised such as moisture and temperature regimes during fruit maturation, and fertilizer application (Chowdhury, 1999). Farmers and crop producer used seed treatment for increasing the seed quality along with yield and to reduce the transmission of pathogens (Khanzada et al., 2002). The purpose of these seed treatments are to control seed-borne pathogens or to protect seeds from soil borne pathogens (Ashley \& Martin, 2003). According to Khanzada et al. (2002) 80-90 percent farmers were used pesticides even after the harvesting seed disinfectant for preventing their harvested crop from post harvesting insect and pests (Borgen, 2004).

Efficacy of seed treatment depends on various factors such as physiological \& physical conditions of the seed, storage conditions, storage time and types of disinfectant used. Therefore producers should be aware about the types of pesticides used and its active ingredients. Information's regarding the effect of moisture contents and proper time of pesticide application in wheat production is in scarcity; therefore present study has been under taken to evaluate the effect of various moist contents on the pesticide application and on various germination components of the wheat crops.

\section{Materials and Methods}

Present study was conducted in the cropping season of 20132014 at the agricultural research station of Ankara University in Ankara Turkey. Seeds of Bezostaja-1 bread wheat variety were used for this study. Two chemical materials viz fungicide and insecticide included Diaconazol (1\%) and Chlorpyrifos ethyl (25\% WP) were collected from local vendor and used either individually or in combination. Study was conducted by split- split plot method in a randomized complete block design with three replications. Three level of seed moisture content viz. $9.5 \%$ (as control), 11 and $12.5 \%$ was considered as main plot; seed moisture content (11 and $12.5 \%$ ) was adjusted by adding water. Storage time $(0,1,2,14$ month) was considered as sub factor (split plot) and pesticide treatment included without pesticide (control), fungicide alone, insecticide alone and combination of both was considered as sub - sub factor (split - split plot). Hundred healthy seeds for each treatment were selected manually by hand picking method. Various growth attributes such as Seed germination, Coefficient of velocity of germination (CVG), Germination rate (GI), Germination rate index (GRI), Coleoptile length, Root length, plantlet fresh and dry weight was calculated according to method given by Sharafizad et al.(2013). In order to measured detail (CVG, GI, and GRI):CVG is characterized by speed and acceleration of germination and calculated from the following equation (Maguire, 1962).

$C V G=G 1+G 2+G 3+\ldots G n /(1 \times G 1)+(2 \times G 2)+(3 \times G 3)+\ldots$ $(N \times G n)$

Whereas G1-Gn: the number of germinations since the first day until the last day.

Germination Index (GI) and germination rate index (GRI) was calculated by the equation given by Throneberry \& Smith (1955), for this following formula was used.

$G I=(n \times N 1)+(7 \times N 2)+(6 \times N 3)+(5 \times N 4)+\ldots+(1 \times N n)$

Whereas $\mathrm{N}=$ the number of seed germination, $\mathrm{n}=$ day end

$G R I=G 1 / 1+G 2 / 2+G 3 / 3+\ldots G n / n$

Here $\mathrm{Gn}=$ the number of germination seed in $\mathrm{n}$ day, $\mathrm{n}=$ day end

All the collected data were analyzed with SPSS version 19.1 were grouped according to Duncan test and the relationship between characteristics revealed.

\section{Results and Discussion}

Analysis of interaction between moisture content of seed storage time and pesticide applications suggested a significant difference at level $1 \%$ for all the studied germination characteristic and component such as percent seed germination, coefficient of velocity of germination (CVG), germination index (GI), germination rate index (GRI), coleoptiles length, root length, fresh weight and dry weight. 
Further, in case of trilateral interaction between seed storage time, seed moisture content and application of pesticides (fungicides and insecticides) most of the studied trait was reported significant at $1 \%$ while some traits such as GI, GRI, and coleoptile length was reported significant at $5 \%$.

\subsection{Effect of interaction on seed germination}

In seed Germination characteristics, highest germination percentage (99\%) was reported from the treatment containing fungicide with a two-month storage period and 9.5\% seed moisture while the lowest germination percent $(75.33 \%)$ was reported in the combined application of pesticides and herbicide along with one month storage at $12.5 \%$ moisture (Table 2). In general, germination percentage reduced with the increasing storage time and reducing moisture. Govender et al. (2007) reported that corn seed kept in storage for one year under natural conditions can reduce seed germination and this reduction in germination may be because of the infection of pathogenic fungi under storage condition.

Table 1 Analysis of variance study between various interaction such as seed moisture, storage time and pesticide application.

\begin{tabular}{|c|c|c|c|c|c|c|c|c|c|}
\hline \multirow{2}{*}{ Source } & \multirow[b]{2}{*}{ df } & \multirow[b]{2}{*}{ PSG } & \multirow[b]{2}{*}{ CVG } & \multicolumn{5}{|c|}{ Components of germination } & \multirow[b]{2}{*}{$\begin{array}{c}\text { Dry } \\
\text { Weight } \\
\end{array}$} \\
\hline & & & & GI & GRI & $\begin{array}{l}\text { Coleoptil } \\
\text { e Length }\end{array}$ & $\begin{array}{c}\text { Root } \\
\text { length }\end{array}$ & $\begin{array}{c}\text { Fresh } \\
\text { Weight }\end{array}$ & \\
\hline $\mathrm{L}$ & 2 & 4.04 & 2. 06 & 1.93 & 2.14 & 2.06 & 0.44 & 0.09 & 0.66 \\
\hline Seed moisture & 2 & $17.57 *$ & 7.33 & $20.94 * *$ & $21.81 * *$ & $23.29 * *$ & 4.17 & 5.28 & 0.12 \\
\hline Error & 4 & & & & & & & & \\
\hline Storage time & 3 & $18.81 * *$ & $5.76 * *$ & $20.14 * *$ & $22.02 * *$ & $12.01 * *$ & $4.75 *$ & $45.75^{* *}$ & $14.22 * *$ \\
\hline $\begin{array}{l}\text { Seed moisture } \\
\times \text { Storage time }\end{array}$ & 6 & $7.77 * *$ & $7.55 * *$ & $3.53 *$ & $4.81 * *$ & 1.48 & $5.32 * *$ & $24.09 * *$ & $3.64 * *$ \\
\hline Error & 18 & & & & & & & & \\
\hline Pesticide & 3 & $10.99 * *$ & $18.31 * *$ & $3.64 *$ & $4.18 * *$ & $183.24 * *$ & $24.56^{* * *}$ & $3.89 *$ & $6.91 * *$ \\
\hline $\begin{array}{l}\text { Seed moisture } \\
\times \text { pesticide }\end{array}$ & 6 & $4.83 * *$ & $5.21 * *$ & $2.63 *$ & $2.57 *$ & $3.00 *$ & 1.89 & 1.04 & 2.07 \\
\hline $\begin{array}{l}\text { Storage time } \\
\times \text { pesticide } \\
\end{array}$ & 9 & $3.93 * *$ & $6.77 * *$ & 1.50 & 1.89 & $5.38 * *$ & $5.67 * *$ & 1.47 & 1.64 \\
\hline $\begin{array}{l}\text { Seed moisture } \\
\times \text { Storage time } \\
\times \text { pesticide }\end{array}$ & 18 & $7.53 * *$ & $8.34 * *$ & $3.77 * *$ & $4.08 * *$ & $1.98 *$ & $2.31 * *$ & $2.88 * *$ & $2.28 * *$ \\
\hline Error & 72 & & & & & & & & \\
\hline $\begin{array}{l}\text { Coefficient of } \\
\text { Variation } \%\end{array}$ & & 2.49 & 0.31 & 1.83 & 1.95 & 10.96 & 11.47 & 7.08 & 7.07 \\
\hline
\end{tabular}

** Values are significant probability; here CVG (Coefficient of velocity of germination); GI (germination index); GRI (germination rate index); FGP (Final germination percent), RL (root length), FWS (fresh weight seedling), DWS (dry weight seedling) and PSG (Percent seed germination).

Table 2 Effect of pesticide, storage time and moisture content interaction on seed germination.

\begin{tabular}{|c|c|c|c|c|c|}
\hline Treatments & $\begin{array}{l}\text { Percentage } \\
\text { Germination }\end{array}$ & $\begin{array}{c}0 \text { Month } \\
\text { (No Storage ) }\end{array}$ & 1 Month Storage & 2 Months Storage & $\begin{array}{l}14 \text { months } \\
\text { storage }\end{array}$ \\
\hline \multirow[t]{3}{*}{ Free from pesticides } & $12.5 \%$ Moisture & 98.67 & 98.67 & 96.67 & 94.67 \\
\hline & $11 \%$ Moisture & 97.00 & 97.00 & 98.00 & 93.33 \\
\hline & 9.5\% Moisture & 97.00 & 97.00 & 96.00 & 96.00 \\
\hline \multirow[t]{3}{*}{ Only Pesticides } & $12.5 \%$ Moisture & 98.67 & 95.00 & 97.00 & 96.00 \\
\hline & $11 \%$ Moisture & 95.33 & 97.67 & 96.00 & 91.33 \\
\hline & 9.5\% Moisture & 98.00 & 96.00 & 99.00 & 96.00 \\
\hline \multirow[t]{3}{*}{ Only Herbicide } & $12.5 \%$ Moisture & 96.67 & 97.67 & 95.00 & 89.67 \\
\hline & $11 \%$ Moisture & 94.67 & 94.00 & 97.67 & 92.00 \\
\hline & 9.5\% Moisture & 96.00 & 98.67 & 97.00 & 94.33 \\
\hline \multirow{3}{*}{$\begin{array}{l}\text { Herbicide + Pesticide } \\
\text { in combination }\end{array}$} & $12.5 \%$ Moisture & 97.67 & 75.33 & 97.33 & 92.33 \\
\hline & $11 \%$ Moisture & 97.00 & 97.67 & 95.33 & 88.33 \\
\hline & $9.5 \%$ Moisture & 96.67 & 98.00 & 96.00 & 94.33 \\
\hline
\end{tabular}


Table 3 Effect of pesticide, storage time and moisture content interaction on Coefficient of velocity of germination.

\begin{tabular}{|lccccc|}
\hline Treatments & $\begin{array}{c}\text { Percentage } \\
\text { Germination }\end{array}$ & $\begin{array}{c}\text { O Month } \\
\text { (No Storage })\end{array}$ & $\begin{array}{c}\text { 1 Month } \\
\text { Storage }\end{array}$ & $\begin{array}{c}\text { 2 Months } \\
\text { Storage }\end{array}$ & $\begin{array}{c}\text { 14 months } \\
\text { storage }\end{array}$ \\
\hline Free from pesticides & $12.5 \%$ Moisture & 25.01 & 25.00 & 25.09 & 25.01 \\
\cline { 2 - 6 } & $11 \%$ Moisture & 25.00 & 25.05 & 25.01 & 25.14 \\
\cline { 2 - 6 } & $9.5 \%$ Moisture & 25.04 & 25.03 & 25.02 & 25.01 \\
\hline Only Pesticides & $12.5 \%$ Moisture & 25.01 & 25.05 & 25.08 & 25.03 \\
\cline { 2 - 6 } & $11 \%$ Moisture & 25.10 & 25.03 & 25.04 & 25.16 \\
\cline { 2 - 6 } & $9.5 \%$ Moisture & 25.04 & 25.03 & 25.00 & 25.02 \\
\hline Only Herbicide & $12.5 \%$ Moisture & 25.03 & 25.03 & 25.13 & 25.24 \\
\cline { 2 - 6 } & $11 \%$ Moisture & 25.09 & 25.05 & 25.03 & 25.12 \\
\hline $\begin{array}{l}\text { Herbicide + Pesticide in } \\
\text { combination }\end{array}$ & $9.5 \%$ Moisture & 25.04 & 25.03 & 25.05 & 25.03 \\
\cline { 2 - 6 } & $12.5 \%$ Moisture & 25.04 & 25.87 & 25.05 & 25.16 \\
\cline { 2 - 6 } & $11 \%$ Moisture & 25.08 & 25.07 & 25.03 & 25.34 \\
\hline
\end{tabular}

\section{2 Effect of interaction on coefficient of germination velocity}

Coefficient of Germination Velocity was calculated by the formula proposed by Maguire (1962), among various tested highest coefficient of germination velocity was 25.87 and it was reported in the treatment containing combination pesticides at $12.5 \%$ moisture on the one month storage time. This coefficient was immediately (25.34) followed by the combination of pesticides and herbicide along with 11 percent moisture and 14 months storage period. No significant differences was reported among various treatments and this thing indicated that storage time and moisture was not adversely affected the coefficient of germination, although some reduction was reported with storage time and reducing moisture, it seems that the effect of pesticides on short storage times would be increased CVG (Table 3). Results are in agreement with the findings of Sharafizan et al. (2013) those have reported a significant effect of seed priming and drought condition and interaction between these two on germination coefficient. Further, Zare \& Tavili, (2000) compared the seed priming with different levels of salicylic acid and reported a significant effect of these two on coefficient of seed germination, these observation favor the findings of this study.

\subsection{Effect of interaction ongermination index}

At Germination index trait, the highest index value of 1986 was reported in the seeds treated with fungicide after one month storage and at 9.5 percent moisture contents while lowest germination index (1755) was reported in the seed treated by pesticides with 12.5 percent moisture content and one month storage (Table 4). With increasing storage time and decreasing moisture content germination index were found reduced. Study conducted by Clark \& Scott (1982) suggested that seed disinfection by pesticides affect germination index. Similar type of findings was reported by Randhawa et al. (1985).

Table 4 Effect of pesticide, storage time and moisture content interaction on Germination index.

\begin{tabular}{|c|c|c|c|c|c|}
\hline Treatments & Percentage Germination & $\begin{array}{c}0 \text { Month } \\
\text { (No Storage) }\end{array}$ & $\begin{array}{l}1 \text { Month } \\
\text { Storage }\end{array}$ & $\begin{array}{l}2 \text { Months } \\
\text { Storage }\end{array}$ & $\begin{array}{l}14 \text { months } \\
\text { storage }\end{array}$ \\
\hline \multirow[t]{3}{*}{ Free from pesticides } & $12.5 \%$ Moisture & $1977^{\mathrm{a}}$ & $1973^{\mathrm{a}}$ & $1961^{\mathrm{ab}}$ & $1897^{\mathrm{d}}$ \\
\hline & $11 \%$ Moisture & $1940^{b}$ & $1956^{\mathrm{b}}$ & $1964^{\mathrm{ab}}$ & $1911^{\text {cd }}$ \\
\hline & $9.5 \%$ Moisture & $1907^{\text {cd }}$ & $1948^{b}$ & $1928^{\mathrm{bc}}$ & $1924^{\mathrm{c}}$ \\
\hline \multirow[t]{3}{*}{ Only Pesticides } & $12.5 \%$ Moisture & $1977^{\mathrm{a}}$ & $1916^{\mathrm{c}}$ & $1964^{\mathrm{ab}}$ & $1928^{\mathrm{bc}}$ \\
\hline & $11 \%$ Moisture & $1939^{b}$ & $1961^{\mathrm{ab}}$ & $1932^{\mathrm{bc}}$ & $1875^{\mathrm{de}}$ \\
\hline & $9.5 \%$ Moisture & $1972^{\mathrm{a}}$ & $1928^{\mathrm{bc}}$ & $1980^{\mathrm{a}}$ & $1922^{\mathrm{c}}$ \\
\hline \multirow[t]{3}{*}{ Only Herbicide } & $12.5 \%$ Moisture & $1941^{b}$ & $1961^{\mathrm{ab}}$ & $1940^{b}$ & $1865^{\mathrm{e}}$ \\
\hline & $11 \%$ Moisture & $1918^{\mathrm{c}}$ & $1896^{\mathrm{d}}$ & $1961^{\mathrm{ab}}$ & $1876^{\mathrm{de}}$ \\
\hline & $9.5 \%$ Moisture & $1932^{\text {bc }}$ & $1981^{\mathrm{a}}$ & $1956^{\mathrm{ab}}$ & $1895^{\mathrm{d}}$ \\
\hline \multirow{3}{*}{$\begin{array}{l}\text { Herbicide + Pesticide in } \\
\text { combination }\end{array}$} & $12.5 \%$ Moisture & $1962^{\mathrm{ab}}$ & $1755^{\mathrm{f}}$ & $1963^{\mathrm{ab}}$ & $1888^{\mathrm{d}}$ \\
\hline & $11 \%$ Moisture & $1944^{\mathrm{b}}$ & $1915^{c}$ & $1969^{\mathrm{ab}}$ & $1871^{\mathrm{de}}$ \\
\hline & $9.5 \%$ Moisture & $1895^{\mathrm{d}}$ & $1976^{\mathrm{a}}$ & $1948^{\mathrm{b}}$ & $1907^{\text {cd }}$ \\
\hline
\end{tabular}

Value without common letter is showing statistically significant difference 
Table 5 Effect of pesticide, storage time and moisture content interaction on Germination rate index.

\begin{tabular}{|c|c|c|c|c|c|}
\hline Treatments & $\begin{array}{c}\text { Percentage } \\
\text { Germination }\end{array}$ & $\begin{array}{c}\text { 0 Month } \\
\text { (No Storage ) }\end{array}$ & $\begin{array}{l}1 \text { Month } \\
\text { Storage }\end{array}$ & $\begin{array}{l}2 \text { Months } \\
\text { Storage }\end{array}$ & $\begin{array}{c}14 \text { months } \\
\text { storage }\end{array}$ \\
\hline \multirow[t]{3}{*}{ Free from pesticides } & $12.5 \%$ Moisture & $1.030^{\mathrm{a}}$ & $1.027^{\mathrm{ab}}$ & $1.023^{\mathrm{ab}}$ & $0.987^{\mathrm{d}}$ \\
\hline & $11 \%$ Moisture & $1.010^{\mathrm{bc}}$ & $1.020^{\mathrm{b}}$ & $1.023^{\mathrm{ab}}$ & $0.993^{\mathrm{c}}$ \\
\hline & $9.5 \%$ Moisture & $1.017^{\mathrm{b}}$ & $1.017^{\mathrm{b}}$ & $1.003^{\mathrm{bc}}$ & $1.003^{\mathrm{bc}}$ \\
\hline \multirow[t]{3}{*}{ Only Pesticides } & $12.5 \%$ Moisture & $1.030^{\mathrm{a}}$ & $0.993 \mathrm{c}$ & $1.020^{\mathrm{b}}$ & $1.003^{\mathrm{bc}}$ \\
\hline & $11 \%$ Moisture & $1.010^{\mathrm{bc}}$ & $1.020^{\mathrm{b}}$ & $1.007^{\mathrm{bc}}$ & $0.973^{\mathrm{e}}$ \\
\hline & $9.5 \%$ Moisture & $1.027^{\mathrm{ab}}$ & $1.007^{\mathrm{bc}}$ & $1.030^{\mathrm{a}}$ & $1.003^{\mathrm{bc}}$ \\
\hline \multirow[t]{3}{*}{ Only Herbicide } & $12.5 \%$ Moisture & $1.010^{\mathrm{bc}}$ & $1.023^{\mathrm{ab}}$ & $1.010^{\mathrm{bc}}$ & $0.967^{\mathrm{e}}$ \\
\hline & $11 \%$ Moisture & $1.000^{\mathrm{c}}$ & $0.990^{\mathrm{c}}$ & $1.023^{\mathrm{ab}}$ & $0.973^{\mathrm{e}}$ \\
\hline & $9.5 \%$ Moisture & $1.007^{\mathrm{bc}}$ & $1.033^{\mathrm{a}}$ & $1.020^{\mathrm{b}}$ & $0.983^{\mathrm{d}}$ \\
\hline \multirow{3}{*}{$\begin{array}{l}\text { Herbicide }+ \text { Pesticide in } \\
\text { combination }\end{array}$} & $12.5 \%$ Moisture & $1.023^{\mathrm{ab}}$ & 0.897 & $1.023^{\mathrm{ab}}$ & $0.980^{\mathrm{d}}$ \\
\hline & $11 \%$ Moisture & $1.010^{\mathrm{bc}}$ & $1.027^{\mathrm{ab}}$ & $0.997^{\mathrm{c}}$ & $0.970^{\mathrm{e}}$ \\
\hline & $9.5 \%$ Moisture & $1.017^{\mathrm{b}}$ & $1.030^{\mathrm{a}}$ & $1.013^{\mathrm{bc}}$ & $0.990^{\mathrm{c}}$ \\
\hline
\end{tabular}

Value without common letter is showing statistically significant difference

\subsection{Effect of interaction on germination rate index}

Highest rate index (10.03) was reported in the seed stored for one month and treated by fungicide with moisture content 9.5\% while lowest index (0.879) was reported in one month stored seed and treated by fungicide along with pesticide at 12.5 percent moisture (Table 5).Shamsaddin et al. (2007) reported a significant reduction in GRI as a result of seed storage; this storage time reduced the ability of holding moisture in Stocked seeds. Further, Gholinejad (2011) and Tavakolikakhaki \& Beheshti (2010) reported that GRI can be reduced when seeds exposed to various stressor substances. Pesticide is also one of the stressors and it reduced seed's GRI in long-term storage conditions.

3.5 Effect of interaction on coleoptile length
In case of Coleoptile length, maximum coleoptile length (5.44 $\mathrm{cm}$ ) was reported in the seed stored for one month and having 9.5\% moisture and having pesticide treatment. Very little difference was reported in all storage time and all three moisture levels. However, the shortest coleoptile length $(1.33 \mathrm{~cm})$ was reported from the treatment containing pesticides in combination with 1 month storage time and $12.5 \%$ seed moisture content. Further, it can be concluded that the Coleoptile length was significantly affected by the storage time and application of pesticide, combined application of herbicide and pesticide caused maximum reduction in coleoptiles length (Table 6). Findings of present study are in agreement with the findings of Mandani et al. (2011) those have reported that long time storage caused a significant reduction in coleoptile length of wheat. They observed shortest coleoptile length after 14 days of storage under pesticide application and the longest length in the control condition to which no pesticide was applied.

Table 6 Effect of pesticide, storage time and moisture content interaction on Coleoptile Length $(\mathrm{cm})$.

\begin{tabular}{|c|c|c|c|c|c|}
\hline Treatments & Percentage Germination & $\begin{array}{c}0 \text { Month } \\
\text { (No Storage ) }\end{array}$ & $\begin{array}{l}1 \text { Month } \\
\text { Storage }\end{array}$ & $\begin{array}{l}2 \text { Months } \\
\text { Storage }\end{array}$ & $\begin{array}{l}14 \text { months } \\
\text { storage }\end{array}$ \\
\hline \multirow[t]{3}{*}{ Free from pesticides } & $12.5 \%$ Moisture & $5.333^{\mathrm{a}}$ & $5.160^{\mathrm{ab}}$ & $5.167^{\mathrm{ab}}$ & $4.573^{\mathrm{c}}$ \\
\hline & $11 \%$ Moisture & $4.713^{\mathrm{bc}}$ & $4.833^{\mathrm{bc}}$ & $5.120^{\mathrm{ab}}$ & $4.740^{\mathrm{bc}}$ \\
\hline & 9.5\% Moisture & $5.233^{\mathrm{ab}}$ & $5.440^{\mathrm{a}}$ & $5.067^{\mathrm{b}}$ & $4.673^{\mathrm{bc}}$ \\
\hline \multirow[t]{3}{*}{ Only Pesticides } & $12.5 \%$ Moisture & $3.840^{\mathrm{e}}$ & $3.907^{\mathrm{de}}$ & $3.820^{\mathrm{e}}$ & $3.447^{\mathrm{ef}}$ \\
\hline & $11 \%$ Moisture & $4.187^{\mathrm{d}}$ & $3.720^{\mathrm{e}}$ & $3.807^{\mathrm{e}}$ & $3.827^{\mathrm{e}}$ \\
\hline & $9.5 \%$ Moisture & $3.893^{\mathrm{de}}$ & $3.587^{\mathrm{ef}}$ & $4.060^{\mathrm{de}}$ & $4.147^{\mathrm{d}}$ \\
\hline \multirow[t]{3}{*}{ Only Herbicide } & $12.5 \%$ Moisture & $2.480^{\text {gh }}$ & $2.840^{\mathrm{g}}$ & $3.213^{\mathrm{f}}$ & $3.787^{\mathrm{e}}$ \\
\hline & $11 \%$ Moisture & $3.187^{\mathrm{f}}$ & $3.100^{f}$ & $3.507^{\mathrm{ef}}$ & $3.653^{\text {ef }}$ \\
\hline & $9.5 \%$ Moisture & $4.087^{\mathrm{de}}$ & $3.360^{\mathrm{f}}$ & $4.273^{\mathrm{d}}$ & $3.907^{\mathrm{de}}$ \\
\hline \multirow{3}{*}{$\begin{array}{l}\text { Herbicide + Pesticide in } \\
\text { combination }\end{array}$} & $12.5 \%$ Moisture & $2.367^{\text {gh }}$ & $1.333^{\mathrm{h}}$ & $2.540^{\mathrm{gh}}$ & $3.460^{\mathrm{ef}}$ \\
\hline & $11 \%$ Moisture & $2.473^{\text {gh }}$ & $2.760^{\mathrm{g}}$ & $2.853^{\mathrm{g}}$ & $3.273^{\mathrm{f}}$ \\
\hline & $9.5 \%$ Moisture & $3.047^{\mathrm{f}}$ & $2.360^{\text {gh }}$ & $3.560^{\mathrm{ef}}$ & $3.347^{\mathrm{f}}$ \\
\hline
\end{tabular}

Value without common letter is showing statistically significant difference 
Table 7 The effect of pesticide, storage time and moisture content interaction on root length $(\mathrm{cm})$.

\begin{tabular}{|c|c|c|c|c|c|}
\hline Treatments & $\begin{array}{l}\text { Percentage } \\
\text { Germination }\end{array}$ & $\begin{array}{c}0 \text { Month } \\
\text { (No Storage ) }\end{array}$ & $\begin{array}{l}1 \text { Month } \\
\text { Storage }\end{array}$ & $\begin{array}{l}2 \text { Months } \\
\text { Storage }\end{array}$ & $\begin{array}{l}14 \text { months } \\
\text { storage }\end{array}$ \\
\hline \multirow[t]{3}{*}{ Free from pesticides } & $12.5 \%$ Moisture & $10.73^{\mathrm{d}}$ & $9.847^{\mathrm{de}}$ & $10.20^{\mathrm{d}}$ & $9.320^{\mathrm{e}}$ \\
\hline & $11 \%$ Moisture & $11.08^{\mathrm{c}}$ & $10.49^{\mathrm{d}}$ & $12.45^{\mathrm{b}}$ & $8.800^{\mathrm{ef}}$ \\
\hline & $9.5 \%$ Moisture & $11.37^{\mathrm{c}}$ & $11.19^{\mathrm{c}}$ & $11.22^{\mathrm{c}}$ & $9.567^{\mathrm{e}}$ \\
\hline \multirow[t]{3}{*}{ Only Pesticides } & $12.5 \%$ Moisture & $7.940^{\mathrm{f}}$ & $7.953^{\mathrm{f}}$ & $8.440^{\text {ef }}$ & $9.860^{\mathrm{de}}$ \\
\hline & $11 \%$ Moisture & $8.093^{\mathrm{f}}$ & $8.893^{\mathrm{ef}}$ & $9.447^{\mathrm{e}}$ & $8.240^{\mathrm{f}}$ \\
\hline & $9.5 \%$ Moisture & $10.56^{\mathrm{d}}$ & $8.333^{\mathrm{f}}$ & $9.013^{\text {ef }}$ & $9.893^{\mathrm{de}}$ \\
\hline \multirow[t]{3}{*}{ Only Herbicide } & $12.5 \%$ Moisture & $11.11^{\mathrm{c}}$ & $10.50^{\mathrm{d}}$ & $11.42^{\mathrm{c}}$ & $10.09^{\mathrm{de}}$ \\
\hline & $11 \%$ Moisture & $10.43^{\mathrm{d}}$ & $11.17^{\mathrm{c}}$ & $10.43^{\mathrm{d}}$ & $9.907^{\mathrm{de}}$ \\
\hline & $9.5 \%$ Moisture & $11.33^{\mathrm{c}}$ & $13.07^{\mathrm{a}}$ & $11.37^{\mathrm{c}}$ & $8.853^{\text {ef }}$ \\
\hline \multirow{3}{*}{$\begin{array}{l}\text { Herbicide + Pesticide in } \\
\text { combination }\end{array}$} & $12.5 \%$ Moisture & $9.613^{\mathrm{e}}$ & $3.507^{\mathrm{g}}$ & $9.400^{\mathrm{e}}$ & $9.960^{\mathrm{de}}$ \\
\hline & $11 \%$ Moisture & $9.613^{\mathrm{e}}$ & $9.660^{\mathrm{e}}$ & $9.780^{\mathrm{de}}$ & $10.19^{\mathrm{d}}$ \\
\hline & $9.5 \%$ Moisture & $9.867^{\mathrm{de}}$ & $9.600^{\mathrm{e}}$ & $9.880^{\mathrm{de}}$ & $10.20^{\mathrm{d}}$ \\
\hline
\end{tabular}

Value without common letter is showing statistically significant difference

3.6 Effect of interaction on root length seedling

Trends of root length show similarity with the trends observed in coleoptile length, maximum root length $(13.07 \mathrm{~cm})$ was reported in the seed having $9.5 \%$ moisture content and stored for 1 month along with treated by herbicides. Seed with 11 percent moisture without pesticide application and stored for two months follow the highest root length. The lowest root length $(3.51 \mathrm{~cm})$ was reported in the seed stored for one month and treated with pesticides combination at $12.5 \%$ moisture (Table 7). Results are in agreement with the findings Mandani et al. (2011) reported increase in the length of root and seedling establishment under the influence of seed size and percentage of material content announced; however, at longterm maintenance seed weight is diminished. Mohammadi (2008) reported that the percentage of normal root in Glycine max reduced when the storage time was increased.
3.7 Effect of interaction on fresh weights seedling

Highest average fresh weight $1.90 \mathrm{~g}$ was reported from the treatment with herbicide at 1 month storage in $9.5 \%$ seed moisture content. Seed with $9.5 \%$ moisture and without storage period and pesticide have $1.86 \mathrm{~g}$ fresh weigh which is not significantly different from the highest fresh weight showing treatments. Minimum fresh weight of seedlings $(1.24 \mathrm{~g})$ has been identified with the combination of pesticides and herbicides during the 1months maintenance of the $12.5 \%$ seed moisture content (Table 8). With the storage time fresh weight reduced and after 14 months storage least fresh weight was reported for all treatments. Fresh weight at the beginning of the germination is affected by the vigor of seeds (Mathews \& KhajeHosseini, 2006). The vigor is less favorable with increasing storage period or storage conditions Esmailzadeh et al. (2008).

Table 8 Effect of pesticide, storage time and moisture content interaction on fresh weight $(\mathrm{g})$.

\begin{tabular}{|c|c|c|c|c|c|}
\hline Treatments & Percentage Germination & $\begin{array}{c}0 \text { Month } \\
\text { (No Storage ) }\end{array}$ & $\begin{array}{l}1 \text { Month } \\
\text { Storage }\end{array}$ & $\begin{array}{l}2 \text { Months } \\
\text { Storage }\end{array}$ & $\begin{array}{l}14 \text { months } \\
\text { storage }\end{array}$ \\
\hline \multirow[t]{3}{*}{ Free from pesticides } & $12.5 \%$ Moisture & $1.640^{\mathrm{cd}}$ & $1.753^{\mathrm{bc}}$ & $1.673^{\mathrm{cd}}$ & $1.573^{\mathrm{d}}$ \\
\hline & $11 \%$ Moisture & $1.683^{\mathrm{c}}$ & $1.837^{\mathrm{ab}}$ & $1.690^{\mathrm{c}}$ & $1.747^{\mathrm{bc}}$ \\
\hline & $9.5 \%$ Moisture & $1.867^{\mathrm{a}}$ & $1.723^{\mathrm{bc}}$ & $1.717^{\mathrm{bc}}$ & $1.720^{\mathrm{bc}}$ \\
\hline \multirow[t]{3}{*}{ Only Pesticides } & $12.5 \%$ Moisture & $1.623^{\text {cd }}$ & $1.790^{\mathrm{b}}$ & $1.603^{\mathrm{d}}$ & $1.570^{\mathrm{d}}$ \\
\hline & $11 \%$ Moisture & $1.577^{\mathrm{d}}$ & $1.823^{\mathrm{ab}}$ & $1.700^{\mathrm{c}}$ & $1.557 \mathrm{~d}$ \\
\hline & $9.5 \%$ Moisture & $1.837^{\mathrm{ab}}$ & $1.723^{\mathrm{bc}}$ & $1.680^{\mathrm{c}}$ & $1.643^{\mathrm{cd}}$ \\
\hline \multirow[t]{3}{*}{ Only Herbicide } & $12.5 \%$ Moisture & $1.760^{\mathrm{bc}}$ & $1.810^{\mathrm{ab}}$ & $1.617^{\mathrm{cd}}$ & $1.730^{\mathrm{bc}}$ \\
\hline & $11 \%$ Moisture & $1.723^{\mathrm{bc}}$ & $1.837^{\mathrm{ab}}$ & $1.727^{\mathrm{bc}}$ & $1.447^{\mathrm{e}}$ \\
\hline & $9.5 \%$ Moisture & $1.830^{\mathrm{ab}}$ & $1.903^{\mathrm{a}}$ & $1.893^{\mathrm{a}}$ & $1.583^{\mathrm{d}}$ \\
\hline \multirow{3}{*}{$\begin{array}{l}\text { Herbicide + Pesticide in } \\
\text { combination }\end{array}$} & $12.5 \%$ Moisture & $1.723^{\mathrm{bc}}$ & $1.240^{\mathrm{f}}$ & $1.700^{\mathrm{bc}}$ & $1.630^{\mathrm{cd}}$ \\
\hline & $11 \%$ Moisture & $1.587^{\mathrm{d}}$ & $1.813^{\mathrm{ab}}$ & $1.750^{\mathrm{bc}}$ & $1.557^{\mathrm{d}}$ \\
\hline & $9.5 \%$ Moisture & $1.700^{\mathrm{c}}$ & $1.747^{\mathrm{bc}}$ & $1.650^{\mathrm{cd}}$ & $1.717^{\mathrm{bc}}$ \\
\hline
\end{tabular}

Value without common letter is showing statistically significant difference 
Table 9 The effect of pesticide, storage time and moisture content interaction on Dry weight $(\mathrm{g})$.

\begin{tabular}{|c|c|c|c|c|c|}
\hline Treatments & $\begin{array}{l}\text { Percentage } \\
\text { Germination }\end{array}$ & $\begin{array}{c}0 \text { Month } \\
\text { (No Storage ) }\end{array}$ & $\begin{array}{l}1 \text { Month } \\
\text { Storage }\end{array}$ & $\begin{array}{l}2 \text { Months } \\
\text { Storage }\end{array}$ & $\begin{array}{l}14 \text { months } \\
\text { storage }\end{array}$ \\
\hline \multirow[t]{3}{*}{ Free from pesticides } & $12.5 \%$ Moisture & $0.2467^{\mathrm{d}}$ & $0.2467^{\mathrm{d}}$ & $0.3000^{\mathrm{b}}$ & $0.2067^{\mathrm{e}}$ \\
\hline & $11 \%$ Moisture & $0.2667^{\mathrm{c}}$ & $0.2600^{\mathrm{c}}$ & $0.2867^{\mathrm{bc}}$ & $0.2300^{\mathrm{de}}$ \\
\hline & 9.5\% Moisture & $0.2733^{\mathrm{c}}$ & $0.2733^{\mathrm{c}}$ & $0.2500^{\mathrm{cd}}$ & $0.2633^{\mathrm{c}}$ \\
\hline \multirow[t]{3}{*}{ Only Pesticides } & $12.5 \%$ Moisture & $0.2400^{\mathrm{d}}$ & $0.2733^{\mathrm{c}}$ & $0.2833^{\mathrm{bc}}$ & $0.2667^{\mathrm{c}}$ \\
\hline & $11 \%$ Moisture & $0.2467^{\mathrm{d}}$ & $0.3000^{\mathrm{b}}$ & $0.2833^{\mathrm{bc}}$ & $0.2700^{\mathrm{c}}$ \\
\hline & $9.5 \%$ Moisture & $0.3100^{\mathrm{b}}$ & $0.3133^{\mathrm{b}}$ & $0.2600^{\mathrm{c}}$ & $0.2367^{\mathrm{de}}$ \\
\hline \multirow[t]{3}{*}{ Only Herbicide } & $12.5 \%$ Moisture & $0.2633^{\mathrm{c}}$ & $0.2567^{\mathrm{cd}}$ & $0.3033^{\mathrm{b}}$ & $0.2467^{\mathrm{d}}$ \\
\hline & $11 \%$ Moisture & $0.2800^{\mathrm{bc}}$ & $0.2833^{\mathrm{bc}}$ & $0.2800^{\mathrm{bc}}$ & $0.2467^{\mathrm{d}}$ \\
\hline & $9.5 \%$ Moisture & $0.2467^{\mathrm{d}}$ & $0.2800^{\mathrm{bc}}$ & $0.2833^{\mathrm{bc}}$ & $0.2667^{\mathrm{c}}$ \\
\hline \multirow{3}{*}{$\begin{array}{l}\text { Herbicide }+ \text { Pesticide in } \\
\text { combination }\end{array}$} & $12.5 \%$ Moisture & $0.2867^{\mathrm{bc}}$ & $0.3733^{\mathrm{a}}$ & $0.2967^{\mathrm{bc}}$ & $0.2733^{\mathrm{c}}$ \\
\hline & $11 \%$ Moisture & $0.2733^{\mathrm{c}}$ & $0.2900^{\mathrm{bc}}$ & $0.2700^{\mathrm{c}}$ & $0.3133^{\mathrm{b}}$ \\
\hline & $9.5 \%$ Moisture & $0.2833^{\mathrm{bc}}$ & $0.2767^{\mathrm{c}}$ & $0.2700^{c}$ & $0.2600^{c}$ \\
\hline
\end{tabular}

Value without common letter is showing statistically significant difference

\subsection{Effect of interaction on dry weight seedling}

The highest value in dry weight was $0.373 \mathrm{~g}$ and it was reported from the treatment containing pesticide and herbicide combination along with one month stored seed with $12.5 \%$ seed moisture, this was followed by the 14 month storage with 11 percent moisture and one month storage with 9.5 percent moisture in combination with pesticide and herbicides. . The minimum dry weight is $0.207 \mathrm{~g}$ was reported in the seed stored for 14 months and moisture maintain on $12.5 \%$ without pesticide content (Table 9). Soltani et al. (2007) reported that extended storage time significantly increases the start and end time of seed germination. In addition, increasing storage period decreases dry seedling weight (Mandani et al., 2011).

Overall result of this study suggested that various traits such as germination percentage, germination index, fresh weight, and root length show highest efficiency on one or two moth storage at 9.5 percent moisture content and herbicide application. While the same traits show minimum efficacy on 12.5 percent moisture and combined application of pesticide and herbicide for the same duration. Various evidences show that the best treatment for germination of wheat seeds is disinfection by fungicides for a shorter period of one to two months in the standard moisture content (9.5\%). At other tests conducted by Clark \& Scott (1982) reported that treatment of wheat seed with fungicide was effective on germination rate and seed germination while the same study suggested that the treatment of the seed with fungicides have negative impact and it is not only reducing the seed germination but also reducing the efficiency. In present study, some studied traits such as CVG and dry weight of seedlings, show highest efficiency on one month storage at $12.5 \%$ moisture and combination of pesticides (fungicide + insecticide) content. Similar type of observation was reported by Tavakolikakhaki \& Beheshti (2010). Further, according to Clark \& Scot (1982) the effects of fungicides on and seedling dry weight were not significant. Tavakolikakhaki \& Beheshti (2010) studied the effect of storage time on seed germination, seedling dry weight, shoot length, root length and root to shoot ratio and reported a significant differences among these traits. The results showed that the seed germination percentage, germination rate and other factors measured in seedling cannot predicted under laboratory conditions. However, based on this experimental results we cannot concluded on the non-use of pesticides, fungicides or insecticides for the seed treatment but it can be conclude use of these pesticides can not necessarily increase the percentage and rate of germination of wheat. However, the interaction between the type of fungicide and seed moisture content and storage time affect the characteristics of different pesticides.

\section{Conflict of interest}

Authors would hereby like to declare that there is no conflict of interests that could possibly arise.

\section{References}

Ashley RO, Martin G (2003) spring wheat seed treatment demonstration. Annual Report Agronomy Section Dickinson Research Extension Center. Available at http://www. ag.ndsu.nodak.edu/dickinso/research/2003/agron03i.htm access on 12th December, 2015.

Borgen A (2004) Organic seed treatment to control common bunt (Tilletiatritici) in wheat. Seed Testing International 128: 8-9. Available on http://orgprints.org/4909/1/4909.pdf access on 12th December, 2015.

Chowdhury MA, Mahmood N, Rashid TR, Khaliq I (1999) Effect of leaf removal on grain yield and its components in spring wheat. Rachis (ICARDA), Barley and Wheat Newsletter 18: 75-78. 
Clark SM, Scott DJ (1982) Effects of carboxin, benomyl and captan on the germination of wheat during the Post-harvest dormancy period (1982). Seed Science and Technology 10: 8794.

Dupriez H, De Leener P (1989) African Gardens and Orchards: Growing Vegetables and Fruits. Macmillan Publishers Ltd., London, Pp. 333.

Esmailzadeh J, Aharizadeh S, Dabagh A, Tabataba-Vakili H (2008) Effect of seed deterioration seed storage Germination Percentage and seedling growth of two corn hybrids. Proceeding of the international congress of agronomy, society for agronomy and plant breeding, Karaj, Iran 6:216 - 222.

Gholinejad E (2011) Effect of salutary on germination component in wheat genotypes. Seed Science and Technology journal.

Gliessman SR (2000) Agroecology: Ecological Processes in Sustainable Agriculture. CRC Press, Boca Raton, Florida Pp.317.

Govender V, Aveling TAS, Kritzinger Q (2007) The effect of traditional storage methods on germination and vigour of maize (Zea mays L.) from northern KwaZulu-Natal and southern Mozambique. South African Journal of Botany 74: 190-196. doi:10.1016/j.sajb.2007.10.006.

Khanzada KA, Aslam Rajput M, Sarwar Shah G, MubeenLodhi A, Farrakh Mehboob (2002) Effect of Seed Dressing Fungicides for the Control of Seedborne Mycoflora of Wheat. Asian Journal of Plant Sciences 1: 441-444. DOI: 10.3923/ajps.2002.441.444.

Lu YC, Terry CK (1995) Implication of sustainable agriculture for the world food situation. Food Reviews International 11: 255-280. DOI:10.1080/87559129509541042.

Maguire JD (1962) Seed of germination aid in selection and evaluation for seedling emergence and vigor. Crop Science 2: 176-177.

Mandani F, RiahiniaSh, Khajehoseyni M (2011) check the storage time and seed size on germination and seed vigor traits of different wheat varieties. Journal of Seed Science and Technology 2:14-24.
Matthews S, Khajeh Hosseini M (2006) Mean germination time as an indicator of emergence performance in soil of seed lots of maize (Zea mays). Seed Science and Technology 34: 339-347.

Mohammadi H (2008) the effect of storage on dynamic of seed storage and seedling growth of soybean. Proceedings of the International Congress of Agronomy, Society for Agronomy and Plant Breeding, Karaj, Iran 8:166 -174.

Nord M, Coleman-Jensen A, Andrews M, Carlson S (2009) Household food security in the United States, Department of Agriculture, Economic Research Service. Washington (DC): U.S. 2010 Nov. Economic Research Report No. ERR-108: 6.

Randhawa HS, Sharma HL, Kaur J, Dhaliwal AS (1985) Effect of fungicides on germination and seed mycoflora on wheat under different storage conditions. Pesticides 2: 36-38.

Shamsaddin Saied M, Farahbakhsh H, MaghsoodiMude AA (2007)

Effects of Salt Stress on Germination, Vegetative Growth and some Physiological Characteristics of Canola. Journal of Water and Soil Science 11: 191-202.

Sharafizad M, Naderi A, Siadat SA, Sakinejad T, Lak S (2013) Effect of Salicylic Acid Pretreatment on Germination of Wheat under Drought Stress. Journal of Agricultural Science 5: 179199. DOI: http://dx.doi.org/10.5539/jas.v5n3p179.

Soltani A, Kamkar B, Galeshi S, AkramGhaderi F (2007) Effect of seed storage on resource depletion and heteroterophic growth of wheat seedling. Iranian Journal of Agricultural Sciences 15: 229-259.

Tavakolikakhaki H, Beheshti A (2010) Wheat seed treatment and seed vigor indices from the perspective of its impact on the environment. Journal of Agro ecology 2:168-174.

Throneberry GO, Smith FG (1955) Relation of respiratory enzymatic activity to corn seed viability. Plant physiology 30 : 337-343.

Zare S, Tavili A (2000) Study effect different level salicylic acid on improvement germination traits fox Mulberry plant under drought and salinity range and natural sources. Iran Bulletin 1: 29-39. 